\title{
El e-learning 2.0: de la tecnología a la metodología
}

\author{
María Carmen Llorente Cejudo \\ Profesora Ayudante del Departamento de Didáctica y Organización Educativa de la Universidad de Sevilla \\ karen@us.es
}

|Fecha presentación: 03/10/2012 | Aceptación: 22/11/2012 |Publicación: 20/12/2012

\begin{abstract}
Resumen
El presente artículo ofrece un marco teórico a través del cual se expone la evolución de los diferentes momentos que Internet ha desarrollado y las posibilidades educativas, a través de la evolución del e-learning, el análisis crítico en los errores en su aplicación, y la evolución hacia el concepto de e-learning 2.0, a través de herramientas como las redes sociales y los entornos personales de aprendizaje, sus características más significativas así como el potencial educativo en las instituciones de enseñanza superior.
\end{abstract}

Palabras clave: e-learning, web social, redes sociales, entornos personales de aprendizaje

\section{Resum}

Aquest article ofereix un marc teòric a través del qual s'exposa l'evolució dels diferents moments que Internet ha desenvolupat i les possibilitats educatives, a través de l'evolució de l'e learning, l'anàlisi crítica en els errors en la seva aplicació, i la evolució cap al concepte d'e learning 2.0, a través d'eines com les xarxes socials i els entorns personals d'aprenentatge, les seves característiques més significatives així com el potencial educatiu a les institucions d'ensenyament superior

Paraules clau: e-learning, web social, xarxes socials, entorns personals d'aprenentatge

\section{Abstract}

This article provides a theoretical framework through which shows the evolution of the different times that the Internet has developed and the educational possibilities that implies, through the evolution of e-learning, critical analysis on the errors in application, and the move towards the concept of e-learning 2.0, through tools such as social networking and personal learning environments, its most significant features and educational potential that arise when incorporate them into practice in higher education institutions.

Key words: e-learning, social web, social network, personal learning environments 


\section{Ideas iniciales}

No podemos encontrar demasiadas tecnologías que hayan impactado de manera significativa a lo largo de la historia de la educación a las instituciones formativas. Ni de manera general, ni siquiera en las estrategias y metodologías de enseñanza, en las formas en las cuales se relacionan los profesores y los estudiantes, o en la manera en la que se distribuyen los contenidos, entre otros. De estas pocas, podrían destacarse dos que sí lo han hecho: una, sin lugar a dudas, ha sido el libro de texto; y otra, Internet. Con ello se caería en un error si se afirmase que las otras tecnologías no han tenido influencia, pero éstas han sido colaterales, sin realizar un verdadero cambio o transformación de las bases del sistema.

En el caso de Internet, pueden apuntarse dos grandes formas de utilización que han ejercido fuertes consecuencias: el elearning y la web 2.0. En lo que respecta al primero, las definiciones que se han ofrecido han sido muy diversas. Valga como ejemplo de ellas la que aportaban Area y Adell (2009) entendiéndolo como "... es una modalidad de enseñanza-aprendizaje que consiste en el diseño, puesta en práctica y evaluación de un curso o plan formativo, desarrollado a través de redes de ordenadores y que puede definirse como una educación o formación ofrecida a individuos que están geográficamente dispersos o separados, o que interactúan en tiempos diferidos del docente empleando los recursos informáticos y de telecomunicaciones" (Area y Adell, 2009, 391). En el caso de la segunda, Castaño y otros (2008) señalan que éstas se apoyan en aplicaciones que son dinámicas, donde los contenidos se actualizan constantemente, son colaborativas ya que suelen realizarse por grupos de personas, son simples e intuitivas, pueden ser utilizadas sin necesidad de instalar nada en un ordenador ya que la propia web es la plataforma, se ofrecen a través de entornos amigables e interactivos, y en ellas el usuario tiene una fuerte capacidad para gestionar qué, cuándo y cómo publicar.

La incorporación de la web 2.0 al terreno educativo, ha llevado a acuñar el término educación 2.o, que se podría definir como "... aquella que, teniendo en cuenta las nuevas características de los alumnos, y las posibilidades de las nuevas herramientas de comunicación que se han originado en la red de la filosofía de la web 2.0, se plantea una nueva forma de actuar y de replantear el hecho educativo" (Cabero, 2009).

De todo lo apuntado anteriormente, y siguiendo con la marca 2.o, es evidente que de un tiempo a esta parte, está consolidándose en la literatura educativa el término e-learning 2.o, y a él se va a hacer alusión en el presente artículo, abordando lo que puede significar, las diferencias que por oposición tendría con el que podríamos denominar como $e$ learning 1.o, para finalizar reflexionando lo que puede conllevar su aplicación al sistema educativo.

\section{La evolución del e-learning}

Como consecuencia de la propia evolución de la tecnología, por un lado, o por la búsqueda de nuevas formas de poner en funcionamiento las nuevas acciones virtuales formativas por otro, el e-learning ha ido evolucionando desde sus momentos iniciales. Quizás fuese simplemente la necesidad por buscar acciones formativas de más calidad que fueran resolviendo los problemas con que los aplicadores del e-learning se estaban encontrando. Es por ello que, no ha sido una forma de aplicación de la formación virtual estática sino completamente dinámica. Karrer (2007) ha presentado una evolución del e-learning en la que diferencia tres etapas fundamentales, que denomina como: e-learning 1.o, e-learning 1.3, y e-learning 2.o. Las características fundamentales y diferenciadoras las presenta en el cuadro que se ofrece en la Tabla 1.

Como puede observarse, la primera generación estaría formada por la realización de cursos sincrónicos transmitidos a los alumnos a través de un aula virtual, siendo gestionado el proceso a través de una plataforma de formación virtual o LMS. En la segunda se desarrollan también bastantes actividades a través de microcontenidos ofrecidos a los estudiantes, desarrollados por el profesor a través de gestores de contenidos (CLMs). Y la tercera, supone la utilización de herramientas que facilitan la creación de contenidos y su distribución, apoyándose para ello en las herramientas de software social para crear nuevos modelos de interacción.

\begin{tabular}{|l|l|l|l|}
\hline & \multicolumn{1}{|c|}{ e-learning 1.0 } & \multicolumn{1}{c|}{ e-learning 1.3 } & \multicolumn{1}{c|}{ e-learning 2.0 } \\
\hline Componentes principales & $\begin{array}{l}\text { Couseware } \\
\text { LMSs } \\
\text { Herramientas de autor }\end{array}$ & $\begin{array}{l}\text { Referencias híbridas LCMs } \\
\text { Herramientas de autor rápidas } \\
\text { marcadores sociales } \\
\text { Blogs } \\
\text { Aplicaciones } \\
\text { Mash-ups }\end{array}$ \\
\hline Propietario & $\begin{array}{l}\text { De arriba abajo } \\
\text { Unidireccional }\end{array}$ & De arriba a abajo, colaborativo & $\begin{array}{l}\text { De abajo a arriba } \\
\text { Responsabilidad del } \\
\text { pares. }\end{array}$ \\
\hline Tiempo de desarrollo & Largo & Rápido & Ninguno \\
\hline Tamaño del contenido & 6o minutos & 15 minutos & minuto \\
\hline Tiempo de acceso & Antes del acceso al trabajo & In between work & Durante el trabajo \\
\hline Reuniones virtuales & Aula & Intro. En la oficina & Pares, expertos \\
\hline Entrega & Al mismo tiempo & En muchas piezas & Cuando lo necesites \\
\hline Control de acceso & LMS & Email. Internet. & Búsqueda, RSS feed \\
\hline Conductor & Diseñador & Alumno & Trabajador \\
\hline Creador de comentarios & Diseñador & SME & Usuario \\
\hline
\end{tabular}

Tabla 1. Generaciones del e-learning según hizo Karrer (2007). 


\begin{tabular}{|l|l|l|l|}
\hline & \multicolumn{1}{|c|}{ Primera ola } & \multicolumn{1}{c|}{ Segunda ola } & \multicolumn{1}{c|}{ Tercera ola } \\
\hline Plataforma de aprendizaje & Software propietario & Open source & Aprendizaje abierto (web 2.o). \\
\hline Licencia & Cuota de pago & Gratuita con algunas restricciones & Ninguna \\
\hline Se propone como valor & El producto & El servicio & La comunidad \\
\hline $\begin{array}{l}\text { Tipo de producto } \\
\text { dominante }\end{array}$ & LMS & CMS & A determinar \\
\hline Modelo de negocio & $\begin{array}{l}\text { Cuota por licencia de uso } \\
\text { individual }\end{array}$ & Cuota por servicio & Publicidad \\
\hline Centrado en & Admnistradores & Profesionales & Estudiantes \\
\hline Paradigma de aprendizaje & Cumplimiento de tareas & Grupos personalizados & Personalización \\
\hline Resultados claves & Informes & Cursos & Mapas expertos \\
\hline Objetivo prioritario & $\begin{array}{l}\text { Reducción de costes y } \\
\text { mejora cuota de mercado }\end{array}$ & Transferencia de conocimiento & $\begin{array}{l}\text { Establecimiento de redes } \\
\text { sociales }\end{array}$ \\
\hline Rol del instructor & Experto en la materia & Integral & Participativo \\
\hline
\end{tabular}

Tabla 2. Fases en los productos tecnológicos de innovación en el aprendizaje de Atkins (2007) (Adaptado por Castaño, 2009).

También centrado en la significación que ha tenido la tecnología, Adkins (2007) ofrece otra clasificación basada más en la tecnología, a través de la cual abarca desde los momentos iniciales a los actuales, muy marcados por la utilización de las herramientas web 2.0. En la Tabla 2, se ofrece la propuesta realizada por el autor.

También en otros contextos cercanos, diferentes autores han abordado el tema, así el profesor Salinas (2005) distingue tres etapas de desarrollo y evolución de la aplicación del e-learning:

- Un enfoque tecnológico, que puede considerarse de períodos iniciales pero que, en algunos casos, perdura, y que se basa en la idea de que la sofisticación de dicho entorno proporcionará la tan ansiada calidad del proceso enseñanza-aprendizaje.

- El contenido es el rey, representa una segunda perspectiva que, vaticinando el fracaso del enfoque excesivamente tecnológico, ha basado la calidad del proceso en los contenidos y en la representación del conocimiento que estos ofrecen, teniendo en cuenta qué materiales altamente sofisticados proporcionarían la calidad.

- Y finalmente, un enfoque metodológico, que se centra más en el alumno y que, partiendo de criterios pe- dagógicos, basa la calidad en una adecuada combinación, en cada caso, de decisiones que tienen que ver con la tecnología que debe utilizarse, con la función pedagógica que el entorno cumplirá, y con los aspectos de organización del proceso dentro de dicho entorno.

$\mathrm{Al}$ mismo tiempo, la profesora Begoña Gros (2011), de la Universidad Oberta de Cataluña, centrándose en los recursos de aprendizaje movilizados y en la forma en que son diseñados, habla que ha pasado por tres grandes generaciones:

- Primera generación: que viene marcada por la adaptación de los materiales textuales a formatos web (modelo centrado en los materiales).

- Segunda generación: el énfasis se pone en obtener el mejor campus virtual (plataformas y gestores).

- Tercera generación: modelo apoyado en la colaboración y en la flexibilidad (que el estudiante pueda generar conocimiento de forma conjunta. Implica la superación de la metáfora del aula). Se asume que se puede acceder a los materiales a través de diferentes dispositivos, y no sólo a través del ordenador. Desde esta perspectiva se asume la importancia del aprendizaje como un proceso social (Gros, 2011, 15-16).

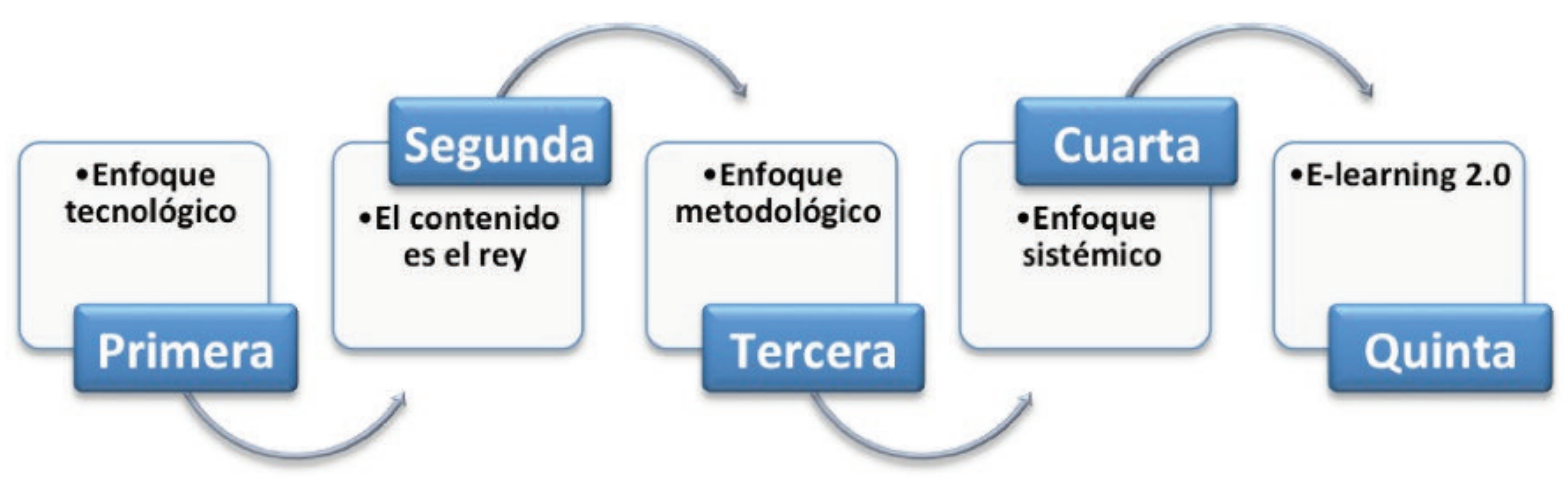

Figura 1. Etapas de evolución del e-learning 
Por otra parte, en un trabajo en desarrollo que será publicado en breve por la editorial Alianza, denominado Tecnologías y medios para la educación en la e-sociedad, coordinado por Aguaded y Cabero (2013), y recogiendo algunos de los planteamientos expuestos, se puede distinguir en la evolución del e-learning diferentes etapas, algunas de las cuales se han simultaneado en el tiempo, como por ejemplo (Figura 1): enfoque tecnológico, el contenido es el rey, enfoque metodológico, un enfoque sistémico, el e-learning 2.o.

En definitiva, y tal como apuntaba Baelo (2009), la adopción del e-learning supone una apuesta por un modelo pedagógico en el que el alumnado toma una mayor responsabilidad en su educación, contribuyendo al desarrollo de la eficiencia en el proceso de enseñanza-aprendizaje, y por ende, a la mejora cualitativa del modelo educativo.

\section{3. ¿Se han cometido errores en la aplicación del e- learning?}

Como se ha apuntado, no se puede dejar de reconocer la significación que el e-learning ha alcanzado en los últimos tiempos, de manera que pocas son las instituciones de formación superior que no hayan establecido planes específicos para su incorporación a la práctica educativa. Algunas veces, porque negarlo, más producto del esnobismo que por la reflexión directa de las posibilidades que podría aportar a la transformación del sistema educativo en la creación de una nueva escenografía comunicativa, que permitiera que profesores y alumnos abordaran el proceso formativo de la enseñanza y el aprendizaje de forma diferente y distinta.

Prueba de ello es que las grandes inversiones se han realizado en tecnologías, que por otra parte se han quedado obsoletas en un breve tiempo, y no en la realización de materiales educativos de calidad para el apoyo del profesorado en su incorporación a la enseñanza, no de forma de un modelo reproductivo de lo que anteriormente estaban realizando, sino mediante acciones formativas innovadoras y colaborativas, o para la transformación de las estructuras organizativas institucionales que dificultan la penetración de una tecnología. Tal penetración no se está llevando a cabo sólo en el mundo de la educación, sino también en la industria y las empresas, como recientemente ha puesto de manifiesto el primer barómetro de e-learning realizado sobre las empresas en Europa (Crossknowledge, 2012). Los errores que se han cometido han sido diversos, muchas veces reflejo de tomar al e-learning como un simple instrumento de ofrecimiento de información al estudiante y repositorio de objetos de aprendizaje, más que como una nueva estrategia de enseñanza que permite flexibilizar la formación independientemente del espacio y el tiempo en el cual estén inmersos los alumnos y el profesor. O bien para crear entornos multimedia, poner a disposición de los alumnos y el profesor diferentes herramientas de comunicación, entre otros. Sin lugar a dudas podemos decir que la penetración del e-learning en las instituciones formativas sólo ha servido para pasar de una Universidad de la fotocopiadora a una Universidad de la impresora. Se ha creído que el simple hecho de trasladar materiales impresos a pdf o html, y su ubicación en la red implicaba el realizar acciones de e-learning o teleformación, y lo único que se estaba realizado era cambiar el mecanismo por el cual la información les era entregada a los estudiantes.

Muchas de las críticas que se le ha efectuado al e-learning han sido consecuencia directa de la importancia que desde los servicios de informática de las instituciones for- mativas se les han dado a la plataforma utilizada, o bien en las grandes posibilidades tecnológicas que debían poseer, o si ellas deberían ser propietarias, libres o auto realizadas. Discusión que, es importante señalar, conlleva más un discurso ideológico que meramente tecnológico. Son famosos los comentarios realizados desde ciertos sectores en insistir que la plataforma Moodle posibilitaba el realizar acciones formativas constructivistas, sin reflexionar respecto a que el modelo de enseñanza es independiente del tipo de plataforma; es más, con todas podemos llevar a cabo acciones formativas bancarias o constructivistas. Por otra parte, como se ha demostrado en diferentes investigaciones, la gran mayoría del profesorado no utiliza en sus acciones formativas virtuales ni la mitad de las posibilidades tecnológicas-instrumentales que le ofrecen las plataformas, e independientemente de sus características, realiza con ellas distintos tipos de acciones metodológicas (Cabero, 2010).

En otro trabajo (Ballesteros y otros, 2010) se hacía referencia a la multitud de servicios que se han creado para facilitar la incorporación del e-learning desde un punto de vista didáctico educativo, desempeñando diferentes funciones, como las siguientes: capacitación del profesorado y el alumnado en el manejo técnico-instrumental de las plataformas de teleformación que se utilizan en las universidades, organización de acciones formativas para facilitar al profesorado la utilización didáctica de estos recursos (aplicación de técnicas y estrategias, tutoría virtual, manejo educativo de las herramientas de comunicación sincrónica y asincrónica, etc.), o producción de material educativo, entre otras.

No es recomendable extenderse aquí en las críticas que se le han realizado a las plataformas, ya que el lector interesado podrá acceder en el trabajo que se encuentra en prensa (Cabero, 2013).

A ellas, pueden incorporarse el aislamiento sociocognitivo en los cuales se ve inmerso el estudiante como consecuencia de la acción formativa creada por el profesor, donde el alumno lo único que debe hacer es bajarse determinados documentos y trabajar de forma memorística con ellos. Aislamiento que puede resolverse mediante la puesta en acción de modalidades b-learning (Llorente, 2009; Castañeda, 2011), y de e-actividades (Cabero y Román, 2007). O también, la ausencia de una formación específica por parte del profesor universitario, que supone una circunstancia que hoy, en la mayoría de los países, se ha convertido en una preocupación, principalmente por los hechos que rodean la nueva universidad que se quiere desarrollar. La formación de los docentes se ha visto, de manera generalizada, como una herramienta además de cómo un arma estratégica para poder llevar a cabo una correcta reforma de los centros universitarios (Marín et al., 2012).

\section{El e-learning 2.0}

Los fallos, limitaciones o errores en su aplicación, está llevando a cabo a diferentes autores a la aplicación de una nueva modalidad del e-learning, que ha venido a denominarse, como se apuntó anteriormente, e-learning 2.o.

Lo primero que se puede señalar, es que el e-learning 2.0 no debe considerarse meramente el sumatorio de las herramientas del e-learning, fundamentalmente la plataforma virtual utilizada, y las herramientas de la web 2.o. Así pues, sin negar dicha combinación de herramientas tecnológicas, el elearning 2.o supone, más bien, un cambio de actitud en cómo abordar y llevar a cabo las acciones formativas soportadas en las redes telemáticas, en las cuales se pasa de modelos mera- 


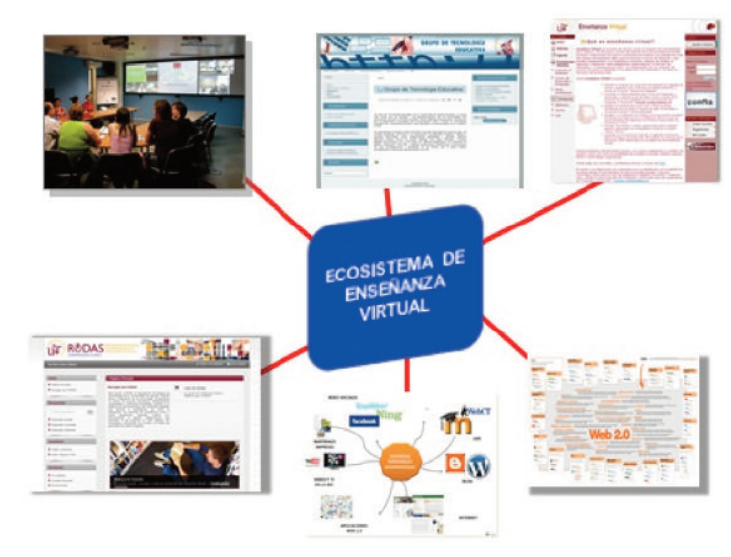

Figura 2. Ecosistema de formación virtual.

mente transmisivos y reproductivos, a modelos donde el alumno se convierte en el centro del escenario de la formación, y el rol del profesor se transforma de transmisor de información al de diseñador de situaciones medidas de aprendizaje, tanto individuales como grupales, para que los alumnos adquieran determinadas competencias y capacidades previstas para la acción formativa. Como se ha apuntado: "Lo afirmado nos lleva a señalar que por mucho que los LMS incorporen herramientas web 2.0 (blog, wikis, rsss,...), si no se da un cambio de actitud, seguiremos llevando a cabo acciones formativas virtuales meramente tradicionales y transmisoras, por muchas herramientas de la web 2.0 que incorporemos: blog, wikis, RSS,..." (Marín y Reche, 2012; Cabero, 2013).

Desde un punto de vista tecnológico, decir que frente a los tradicionales LMS, en las acciones formativas soportadas bajo la perspectiva del e-learning 2.0, el alumno se encuentra con un verdadero ecosistema virtual (Figura 2), donde tiene disponible desde LMS, entornos personales de aprendizaje (PLE), acceso a contenidos abiertos, repositorios de objetos de aprendizaje o redes sociales, entre otros.

Se trata, por tanto, de crear nuevos escenarios formativos que permitan que los estudiantes puedan tener un rol más activo en su propio proceso de formación virtual. En este e-learning 2.0, diferentes tecnologías alcanzarán un papel clave para que el alumno se informe, comunique y adquiera conocimiento, de manera que tenga las competencias para buscar información (Doogle, Tecnhorati, Delicious,...), organizarla (iGoogle, Delicious,..), producirlas (Google, Doc, Blogger, Prezzi,...), publicarlas (Blog, Wikis, Slideshare, YouTube,...) y comunicarlas (Twitter, Facebook, ....).

En definitiva, son varias las tecnologías que en estos momentos están adquiriendo plena significación: los entornos personales de aprendizaje (Personal Learning Environment, PLE) y las redes sociales. Respecto a los primeros, se están configurando como una de las estrategias más nove- dosas para incorporar las tecnologías de la información a los procesos de enseñanza-aprendizaje. En lo que respecta a su conceptualización, diversos autores (Adell y Castañeda, 2010; Cabero y otros, 2011) apuntan que existen dos corrientes en la definición del PLE: una más centrada en los aspectos tecnológicos -y que centra su atención sobre qué implica la creación de un nuevo entorno tecnológico-, y otra más preocupada por lo que supone una nueva estrategia de aprendizaje. De todas formas, es posible señalar que existe un cierto concierto en asumir que un PLE está compuesto por diferentes herramientas (Atwell, 2007 y 2010; Schaffert y Hilzensauer, 2008; Amine, 2009; Adell y Castañeda, 2010), y que de forma general éstas podrían agruparse en tres tipos básicos: lectura, reflexión y relación.

Independientemente de las dos orientaciones mencionadas, existe simultáneamente un número de autores que se refieren a los PLE como un "enfoque pedagógico para la integración intencional y deliberada de espacios de aprendizaje formales e informales" (Dabbagh \& Kitsantas, 2012, 4). O como también podrían apuntarse, se trata de "un nuevo escenario educativo en el cual los alumnos pueden adquirir diferentes competencias, capacidades, objetivos y contenidos, tanto de la perspectiva formal como informal, siendo ambas igual de interesantes desde nuestro punto de vista, con base en teorías como las del conectivismo o del aprendizaje ubicuo, que parte de la idea de la descentralización del aprendizaje" (Barroso, Cabero y Vázquez, 2012). En definitiva, es posible asumir que un PLE es una recopilación de herramientas establecidas con el propósito de que puedan ser utilizadas por un usuario en función de sus necesidades, destinadas fundamentalmente a la incorporación para su trabajo personal y, por supuesto, para el desarrollo de acciones de aprendizaje (Cabero y otros, 2011).

Si se apunta a algunas de sus ventajas, a modo de síntesis pueden señalarse las siguientes: los alumnos se convierten en unos actores activos en su propio proceso de aprendizaje y llegan a tener una identidad formativa más allá de los contextos tradicionales de aprendizaje; los alumnos adquieren el control y la responsabilidad sobre su propia acción formativa; son fáciles y amigables de construir y manejar, pues tienden a desenvolverse y construirse bajo herramientas web 2.0; pueden poseer una casi ilimitada variedad y funcionalidad de herramientas de comunicación e interacción; el derecho de autor y la reutilización recaen sobre el sujeto -pues él, y no la institución, es dueño de los contenidos y de la información creada y elaborada-; aumento de la presencia social; son entornos abiertos a la interacción y relación con las personas independientemente de su registro oficial en los programas o cursos; se potencia con ellos acciones formativas tanto formales como no formales e informales; y está centrado en el estudiante, es decir, cada alumno elige y utiliza las herramientas que tienen sentido para sus necesidades y circunstancias particulares.

Por lo que se refiere a sus limitaciones y debilidades,

\begin{tabular}{|l|l|}
\hline \multicolumn{1}{|c|}{ e-learning 1.0 } & \multicolumn{1}{c|}{ e-learning 2.0 } \\
\hline Plataforma LMS y LCMS. & Herramientas de la web 2.0. \\
Basado en el profesor. & Basado en el estudiante. \\
El profesor produce. & El profesor valida \\
El estudiante es espectador. & El estudiante es productor. \\
Intercambio con la clase. & Intercambio con la comunidad. \\
\hline
\end{tabular}

Tabla 3. Diferencias entre el e-learning 1.0 y 2.0 (Sbnihi y Eddine, 2010, 4). 
apuntar las siguientes: existe más un desarrollo tecnológico que modelos conceptuales de actuación educativa y formativa; su creación exige de profesores y alumnos con una fuerte capacitación conceptual y tecnológica; y limitado control institucional sobre el proceso y el producto.

Y por lo que respecta a las redes sociales, ya afirmaba Dans (2007) que algo estaba cambiando, cuando hacia referencia a la evolución de la web de segunda mitad de la última década del siglo pasado a la web 2.0, donde el desarrollo se presenta como un ecosistema en Internet en el que los individuos pueden tener tanta importancia como las empresas o los medios de comunicación, un entorno en el que lo importante son las personas.

En lo que respecta a las posibilidades que las redes sociales ofrecen en el ámbito educativo, se pueden sintetizar en las siguientes:

- Contar con un entorno rico y variado, donde podemos utilizar diferentes tipos de recursos y documentos, desde los textuales hasta los visuales y audiovisuales.

- Es un multi-entorno de comunicación, ya que podemos abrir en ella diferentes lugares y espacios para la discusión y el análisis de diferentes problemáticas. Ello facilita su adaptación a diferentes estilos de aprendizaje e inteligencias múltiples de los estudiantes.

- Convierten a profesores y alumnos en emisores y productores de objetos de aprendizaje, lo cual nos lleva a replantearnos el proceso de construcción de conocimientos. - Es un entorno interactivo, en el cual las personas que configuran la red social pueden relacionarse entre ellas, con el profesor, o en la interacción con los diferentes documentos que se hayan ido aportando. Se trata de un espacio no pensado para la reposición de documentos y contenidos, sino para la comunicación entre las personas.

- Son entornos que permiten la comunicación independientemente del espacio y el tiempo en el cual se encuentren ubicados las personas de la comunidad virtual de aprendizaje. Son, por tanto, entornos flexibles para el aprendizaje que facilitan la movilidad virtual de los estudiantes y profesores.

- Pueden ser entornos multiculturales, al poder participar personas de otros contextos, favoreciendo de esta manera una formación multicultural de sus participantes. Ello implica un matiz problematizado que debe ser tenido en cuenta por los participantes de la comunidad virtual de aprendizaje, pues el estar en un mismo espacio tecnológico no significa que se esté en uno mismo cultural, ya que cada uno sigue perteneciendo a su propio entorno cultural, con sus visiones y realidades.

- Son entornos que permiten el control por los estudiantes de su propio proceso de aprendizaje, pues él decide (aunque sólo en cierta media) cuándo participar, en qué momento efectuar el análisis de los documentos, o cómo aportar un documento y en qué formato.

- Al quedar registradas las participaciones de las personas que conforman la CVA se facilita el reflexionar sobre la práctica educativa que han llevado a cabo, su esfuerzo de participación, la calidad de las intervenciones, y el proceso seguido en la construcción del conocimiento.

- Puede utilizarse en todas las disciplinas y para una diversidad de objetivos.

- Su utilización facilita la potenciación de la identidad del alumno y la adquisición de competencias digitales.

- Aumenta la implicación y la motivación del estudiante.
- Permiten la revisión por parte del profesor del proceso seguido para la construcción del conocimiento; es decir, puede ser una herramienta de extraordinario interés no sólo para alcanzar productos cognitivos, sino también para conocer cómo se ha llegado al mismo e identificar errores en el proceso seguido.

Resulta imprescindible tener en cuenta y no olvidar que una herramienta tecnológica por sí misma no va a resolver las necesidades educativas que tengamos con nuestros alumnos en el aula, por eso es necesario apuntar que, incorporar las redes sociales en el ámbito educativo supone tener en cuenta una serie de principios a los que ya, en trabajos anteriores, se hicieron referencia y que se declaraban en los siguientes términos:

- Incorporar una Red Social al aula no supone obtener efectos inmediatos en lo que al aprendizaje de los alumnos se refiere.

- Debe estar basada, fundamentalmente, en los principios del trabajo colaborativo para su mayor rendimiento como recurso educativo.

- El docente tiene a su disposición una enorme cantidad de elementos multimedia a incorporar, los cuales deben responder a un fin educativo.

- Las funciones de guía, dinamizador y orientador deben acaparar la mayor parte del tiempo del docente, por lo que a priori deberá prever posibles pautas de actuación por parte de los estudiantes.

- Al trabajar con la red los alumnos pueden acceder a la diversidad de información, recursos, webs, etc., disponibles en la misma, por lo que el diseño de la tarea deberá contemplar el desarrollo de competencias para la selección y procesamiento de la información disponible (Llorente, 2011).

\section{A modo de conclusión}

Antes de finalizar la exposición, resulta necesario realizar una llamada de atención, para apuntar algunos de los problemas respecto a diferentes problemas con que es posible encontrarse a la hora de poner en funcionamiento, desde un punto de vista educativo, acciones formativas 2.0. El primero de ellos se refiere al alumno, y más concretamente, al nivel de formación que debe poseer el estudiante. Ello es debido a que éste debe estar capacitado para saber trabajar de forma autónoma en su propio proceso de aprendizaje, seleccionando y evaluando los diferentes recursos de aprendizaje que le pueden ser más útiles en el mismo. Por otra parte, debe estar también capacitado para saber trabajar de forma conjunta y colaborativa, lo que implica tener capacidades para la interacción, la negociación y saber aceptar los puntos de vistas ofrecidos por otros.

Independientemente de lo comentado, el alumno debe poseer diferentes competencias tecnológicas en el manejo de herramientas de la web 2.0, tanto para la producción como para la posterior comunicación. Y su alcance debe ser superior a la elaboración de documentos textuales y alcanzar a otros tipos de recursos, tales como los audiovisuales, multimedia y telemáticos.

Pero si el alumno debe transformar sus roles, el docente debe hacerlo también en una serie de direcciones, olvidando el rol tradicionalmente desempeñado de transmisor de información y convertirse en diseñador de situaciones mediadas de aprendizaje; es decir, en la creación de escenarios virtuales, como el que anteriormente se ha comen- 
tado, que favorezca la adquisición de aprendizajes por los estudiantes. Es interesante la apreciación de Prensky (2011, 14) cuando señala lo siguiente: "Pero de nuevo esto es una paradoja porque para tener más éxito en el uso de tecnologías en sus aulas los profesores no necesitan aprender a usarlas ellos mismos (aunque si quieren pueden hacerlo). Lo que los profesores sí necesitan saber es cómo la tecnología puede y debe ser usada por los estudiantes para mejorar su propio aprendizaje“. Y por otra, con la reflexión que establecen Revuelta y Pérez (2009, 114): "Poner en práctica los procesos interactivos en la web 2.0 no ha sido una tarea fácil. Necesitábamos de una novedad pedagógica. Para dar el salto del e-learning al e-learning 2.o se plantea la necesidad de que una corriente pedagógica asome la cabeza al nuevo contexto y de solución, o al menos que fije una postura sólida ante las nuevas demandas".

Quisiéramos finalizar nuestra exposición señalando de nuevo una idea ya expuesta a lo largo del trabajo, y es que el e-learning 2.0 no se produce sólo por el sumatorio de las herramientas del e-learning 1.0 y las del entramado web 2.0, sino que supone un cambio de actitud y una nueva forma de orientar el aprendizaje virtual.

\section{Bibliografía}

Adell, J. \& Castañeda, L. (2010). Los Entornos Personales de Aprendizaje (PLEs): una nueva manera de entender el aprendizaje. En Roig Vila, R. \& Fiorucci, M. (Eds.). Claves para la investigación en innovación y calidad educativas. Alcoy: Marfil - Roma TRE Universita degli studi.

Adkins, S. (2007). Waves of Innovation: From Open Source to Open Learning, http://www.learningcircuits.org/2007/0707adkins.html Fecha de consulta, 20.01.2009

Aguaded, I. y Cabero, J. (cood.) (2013). Tecnologías y medios para la educación en la e-sociedad. Madrid: Alianza (en prensa).

Amine, M. (2009). PLE - PKN, http://mohamedaminechatti.blogspot.com/2009/04/plepkn.html Fecha de consulta, 13.03.2010.

Area, M. \& Adell, J. (2009). E-learning: enseñar y aprender en espacios virtuales. En De Pablos, J. (coord.). Tecnología Educativa, 391-424. Málaga: Aljibe.

Attwell, G. (2007). The Personal Learning Environments the future of eLearning?. eLearning Papers, 2, 1.

Attwell, G. (2010). Supporting Personal Learning in the Workplace.

http://pleconference.citilab.eu/wpcontent/uploads/2010/09/ple2010_submission_66.pdf Fecha de consulta, 02.07.2011.

Baelo, R. (2009). El eLearning. Una respuesta educativa a las demandas de las sociedades del siglo XXI. PixelBit. Revista de Medios y Educación, 35, 87-96.

Ballesteros, C., Cabero, J., Llorente, M.C. y Morales, J.A. (2010). Usos del e-Learning en las universidades andaluzas. Estado de la situación y análisis de buenas prácticas. Pixel-Bit. Revista de Medios y Educación, 37, 7-18.

Cabero, J. (2009). Educación 2.o ¿Marca?. En Castaño, C. (cood.). Web 2.o: El uso de la web en la sociedad del conocimiento. Investigación e implicaciones educativas. Caracas, Universidad Metropolitana.

Cabero, J. (2013). La formación virtual en el nuevo entramado 2.0: el e-learning 2.0. En Aguaded, I. y Cabero, J. (cood.). Tecnologías y medios para la educación en la e-sociedad. Madrid, Alianza (en prensa).

Cabero, J. (coord.) (2010). Usos del elearning en las Universidades Andaluzas: estado de la situación y análisis de buenas prácticas. Sevilla, Grupo de Investigación Didáctica.

Cabero, J. y Román, P. (2007). E-actividades. Un referente básico para la formación en Internet. Sevilla, MAD.

Cabero, J., Marín, V. e Infante, A. (2011). Creación de un entorno personal para el aprendizaje: desarrollo de una experiencia. EDUTEC, Revista Electrónica de Tecnología Educativa, 38. Disponible en:

http://edutec.rediris.es/Revelec2/Revelec38/, Fecha de consulta, 04.03.2012

Castañeda, L. y Sánchez, M. (2010). El mundo enredado. Evolución e historia de las redes sociales. En Castañeda, L. (Coord.). Aprendizaje con redes sociales. Tejidos educativos para los nuevos entornos. Sevilla: Eduforma.

Castañeda, L. (2011). Analizar y entender la enseñanza flexible. Un modelo de análisis de desarrollo curricular. Pixel-Bit. Revista de Medios y Educación, 39, 167-195.

Castaño, C., Maíz, I., Palazio, G. y Villaroel, J. (2008). Prácticas educativas en entornos web 2.o. Madrid: Síntesis.

Crossknowledge (2012). El primer barómetro de e-learning en Europa, CrossKnowledge. Learning for a better life. http://www.crossknowledge.com/es_ES/elearning/m edia-center/publicaciones.html?mictrl=cklgWhitepaper/show\&sid=barometro-elearning-europa, Fecha de consulta, 12.09.2012.

Dans, E. (2007). La empresa y la web 2.0; Harvard Deusto Márketing \& Ventas, 8o. http://www2.edeusto.com/cdrevistasw/hdmv.htm, Fecha de consulta, 20.02.2012

Gros, B. (2011). El modelo educativo basado en la actividad de aprendizaje. En Gros, B. (ed). Evolución y reto de la educación virtual. Construyendo el e-learning del siglo XXI. Barcelona, UOC.

Karrer, T. (2007). Understang ding E-Learning 2.o. http://www.astd.org/LC/2007/0707_karrer.htm, Fecha de consulta 20.01.2012.

Llorente, M.C. (2009). Formación semipresencial apoyada en la red (Blended learning). Diseño de acciones para el aprendizaje. Sevilla: Eduforma.

Llorente, M.C. (2011). De la web tradicional a la web semántica: cambios y aplicación al ámbito educativo. Apertura. Revista de Innovación Educativa, 14, 120-130.

Marín, V. y Reche, E. (2012). Universidad 2.0: Actitudes y aptitudes ante las TIC del alumnado de nuevo ingreso de la Escuela Universitaria de Magisterio de la UCO. Pixel-Bit. Revista de Medios y Educación, 4O, 197-211.

Marín, V., Vázquez, A., Llorente, M.C. y Cabero, J. (2012). La alfabetización digital del docente universitario en el Espacio de Educación Superior. Edutec, Revista Electrónica de Tecnología Educativa, 39. http://edutec.rediris.es/Revelec2/Revelec39/alfabetizacion_digital_d ocente_universitario_EEES.html, Fecha de consulta 03.10.2012.

Prensky, M. (2011). Enseñar a nativos digitales. Madrid: SM. Revuelta, F.I. y Pérez, (2011). Interactividad en los entornos de formación on-line. Barcelona: UOC.

Salinas, J. (2009). Nuevas modalidades de formación: entre los entornos virtuales institucionales y los personales de aprendizaje. En Tejada, J. (coord.), Estrate- 
gias de innovación en la formación para el trabajo.

Madrid: Torrepunta Ediciones.

| Cita recomendada de este artículo

Llorente Cejudo, María Carmen (2012). El e-learning 2.0: de la tecnología a la metodología. @tic. revista d'innovació educativa. ( $\mathrm{n}^{\circ}$ 9). URL.

Fecha de consulta, dd/mm/aaaa. 